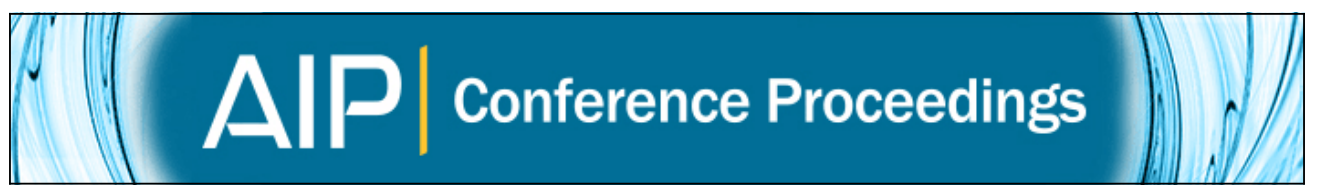

\title{
Elastic scattering with weakly bound projectiles
}

J. M. Figueira, D. Abriola, J. O. Fernández Niello, A. Arazi, O. A. Capurro, G. V. Martí, D. Martínez Heinmann, A. J. Pacheco, J. E. Testoni, E. de Barbará, I. Padrón, P. R. S. Gomes, and J. Lubian

Citation: AIP Conference Proceedings 884, 185 (2007); doi: 10.1063/1.2710577

View online: http://dx.doi.org/10.1063/1.2710577

View Table of Contents: http://scitation.aip.org/content/aip/proceeding/aipcp/884?ver=pdfcov Published by the AIP Publishing

\section{Articles you may be interested in}

Analysis of the elastic scattering measured with a $23.7 \mathrm{MeV} 7 \mathrm{Be}$ beam on a 9 Be target AIP Conf. Proc. 1139, 123 (2009); 10.1063/1.3157792

Elastic Scattering and Reaction Cross Section of the $6 \mathrm{He}+27 \mathrm{Al}$ System Close to the Coulomb Barrier

AIP Conf. Proc. 853, 102 (2006); 10.1063/1.2338362

The effects of deformed projectile in threshold anomaly and fusion reaction AIP Conf. Proc. 610, 599 (2002); 10.1063/1.1469998

Analysis of the elastic $6 \mathrm{Li}+12 \mathrm{C}$ scattering: Energy dependence, "abnormal dispersion" and dynamic polarization potential

AIP Conf. Proc. 455, 510 (1998); 10.1063/1.57251

The description of exotic nuclei elastic scattering in the framework of glauber model with noneikonal corrections

AIP Conf. Proc. 455, 331 (1998); 10.1063/1.57230 


\title{
Elastic scattering with weakly bound projectiles
}

\author{
J. M. Figueira*,†, D. Abriola*, J. O. Fernández Niello*,**, A. Arazi*, O. A. \\ Capurro*, G. V. Martí, D. Martínez Heinmann*, A. J. Pacheco*, J. E. \\ Testoni*, E. de Barbará*, I. Padrón ${ }^{\ddagger}$ P. R. S. Gomes ${ }^{\ddagger}$ and J. Lubian \\ ${ }^{*}$ Laboratorio TANDAR, Comisión Nacional de Energía Atómica, Av. General Paz 1499, \\ 1650 San Martín, Buenos Aires, Argentina. \\ ${ }^{\dagger}$ Departamento de Física, FCEyN, Universidad de Buenos Aires, Ciudad Universitaria, \\ Pabellón 1, 1428 Buenos Aires, Argentina \\ ** Escuela de Ciencia y Tecnología, Universidad Nacional de General San Martín, \\ Martín de Irigoyen 3100, 1650 San Martín, Buenos Aires, Argentina. \\ ${ }^{\sharp}$ Instituto de Física, Universidade Federal Fluminense, Avenida Litoranea s/n, Gragoatá,
} Niterói, R. J., 24210-340, Brazil.

\begin{abstract}
Possible effects of the break-up channel on the elastic scattering threshold anomaly has been investigated. We used the weakly bound ${ }^{6,7} \mathrm{Li}$ nuclei, which is known to undergo break-up, as projectiles in order to study the elastic scattering on a ${ }^{27} \mathrm{Al}$ target. In this contribution we present preliminary results of these experiments, which were analyzed in terms of the Optical Model and compared with other elastic scattering data using weakly bound nuclei as projectile.
\end{abstract}

Keywords: Elastic scattering, ${ }^{6,7} \mathrm{Li}$ projectiles; Deduced optical model parameters; Threshold anomaly; Weakly bound nuclei.

PACS: 25.70.Bc; 24.10.Ht, 27.20.+n

\section{INTRODUCTION}

The elastic scattering close to the Coulomb barrier of tightly bound nuclei described by the Optical Model shows a rapid variation of both the real and imaginary parts of the potential as a function of energy. This variation is consistent with a dispersion relation and has been named the "threshold anomaly" [1].

The variation of the imaginary part of the potential has been explained as a closing of the quasi-elastic channels when the energy approaches the Coulomb barrier. When one of the participants is weakly bound, there is a possibility of having a break-up channel that does not close so fast in the vicinity of the Coulomb barrier. This effect has been proposed as a possible explanation of the absence of the "normal threshold anomaly" for ${ }^{6,7} \mathrm{Li}+{ }^{28} \mathrm{Si}[2,3]$.

In the present work the elastic scattering of two weakly bound projectiles against an aluminum target is studied at energies close to the Coulomb Barrier to ascertain whether the "threshold anomaly" is present or not. The fusion cross section for these systems measured close to the Coulomb barrier is presented elsewhere in these proceedings [4] . The ultimate goal is to describe fusion and quasi-elastic data within the same model.

CP884, VI Latin American Symposium on Nuclear Physics and Applications,

edited by O. Civitarese, C. Dorso, G. García Bermúdez, A. J. Kreiner, A. J. Pacheco, and N. N. Scoccola (C) 2007 American Institute of Physics 978-0-7354-0388-8/07/\$23.00 


\section{EXPERIMENTAL SETUP}

The experiments were performed at the 20 UD tandem accelerator of the Tandar Laboratory in Buenos Aires. We used ${ }^{6} \mathrm{Li}$ and enriched ${ }^{7} \mathrm{Li}$ beams, being their $Q$-value for alpha particle break-up $-1.47 \mathrm{MeV}$ and $-2.47 \mathrm{MeV}$, respectively. The target was a ${ }^{27} \mathrm{Al}$ foil $70 \mu \mathrm{g} / \mathrm{cm}^{2}$ thick. For the ${ }^{6} \mathrm{Li}$ beam we measured the elastic scattering at five different bombarding energies $(7,8,10,12$, and $18 \mathrm{MeV})$, whereas for the ${ }^{7} \mathrm{Li}$ beam the measured energies were $6,7,8,9,10,11,12,14,16$, and $18 \mathrm{MeV}$. The nominal Coulomb barrier for these systems is around $8 \mathrm{MeV}$.

A set of eight Silicon detectors were used to measure the elastic scattering cross sections at different angles. Angular distributions were determined in steps of $2^{\circ}$ to $5^{\circ}$ depending on the energy and angular range. The angular resolution of each detector was 0.5 degrees and their energy resolution ranged from 0.5 to $1 \%$. Normalization was achieved by using an electron suppressed Faraday cup and a monitor detector at 15 degrees.

\section{RESULTS AND OPTICAL MODEL ANALYSIS}

In this work the elastic scattering in the ${ }^{7} \mathrm{Li}+{ }^{27} \mathrm{Al}$ system has been adjusted using the code Ptolemy [5]. An energy-independent potential that reproduces the data has been found and is shown in Table 1. The potential has an imaginary part composed by two terms. A volume part with a Woods-Saxon shape of parameters W (depth), $\mathrm{r}_{i 0}$ (reduced radius) and $a_{i}$ (diffusivity), to take into account the fusion reactions, and a surface part, which is the derivative of a Woods-Saxon shape of parameters WS, $\mathrm{r}_{s i 0}$ and $a_{s i}$, to take into account the quasi elastic reactions [6,7]. The real part is a Woods-Saxon potential of parameters $\mathrm{V}, \mathrm{r}_{0}$ and $a$. The fit results and the experimental angular distributions are shown in Figure 1.

TABLE 1. Potential parameters used for the energy-independent Optical Model fits.

\begin{tabular}{ccccccccc}
\hline $\begin{array}{c}\mathrm{V} \\
(\mathrm{MeV})\end{array}$ & $\begin{array}{c}\mathrm{r}_{0} \\
(\mathrm{fm})\end{array}$ & $\begin{array}{c}a \\
(\mathrm{fm})\end{array}$ & $\begin{array}{c}\mathrm{W} \\
(\mathrm{MeV})\end{array}$ & $\begin{array}{c}\mathrm{r}_{i 0} \\
(\mathrm{fm})\end{array}$ & $\begin{array}{c}a_{i} \\
(\mathrm{fm})\end{array}$ & $\begin{array}{c}\mathrm{WS} \\
(\mathrm{MeV})\end{array}$ & $\begin{array}{c}\mathrm{r}_{s i 0} \\
(\mathrm{fm})\end{array}$ & $\begin{array}{c}a_{s i} \\
(\mathrm{fm})\end{array}$ \\
\hline 9.86 & 1.12 & 0.904 & 1.31 & 1.09 & 0.372 & 2.21 & 1.35 & 0.646 \\
\hline
\end{tabular}

To explore for a possible energy-dependence a new parameter search was performed in which the depths of the real and imaginary parts of the potential were allowed to change independently at each energy. For this study, all the other "geometric" parameters were kept constant, avoiding in this way the need of calculating the potentials at the "sensitivity radius". The results for this energy-dependent potential are also shown in Figure 1 . The global $\chi^{2} /$ point corresponding to this fit is 2.34 , whereas for the energyindependent fit it takes the value of 3.89. As can be seen, it represents only a slight improvement to the adjustment of the data compared to the energy-independent value.

The depths of the real and imaginary parts of the energy-dependent potential, $\mathrm{V}$ and WS, respectively (see Figure 2), do not show the "normal threshold anomaly". That is, 


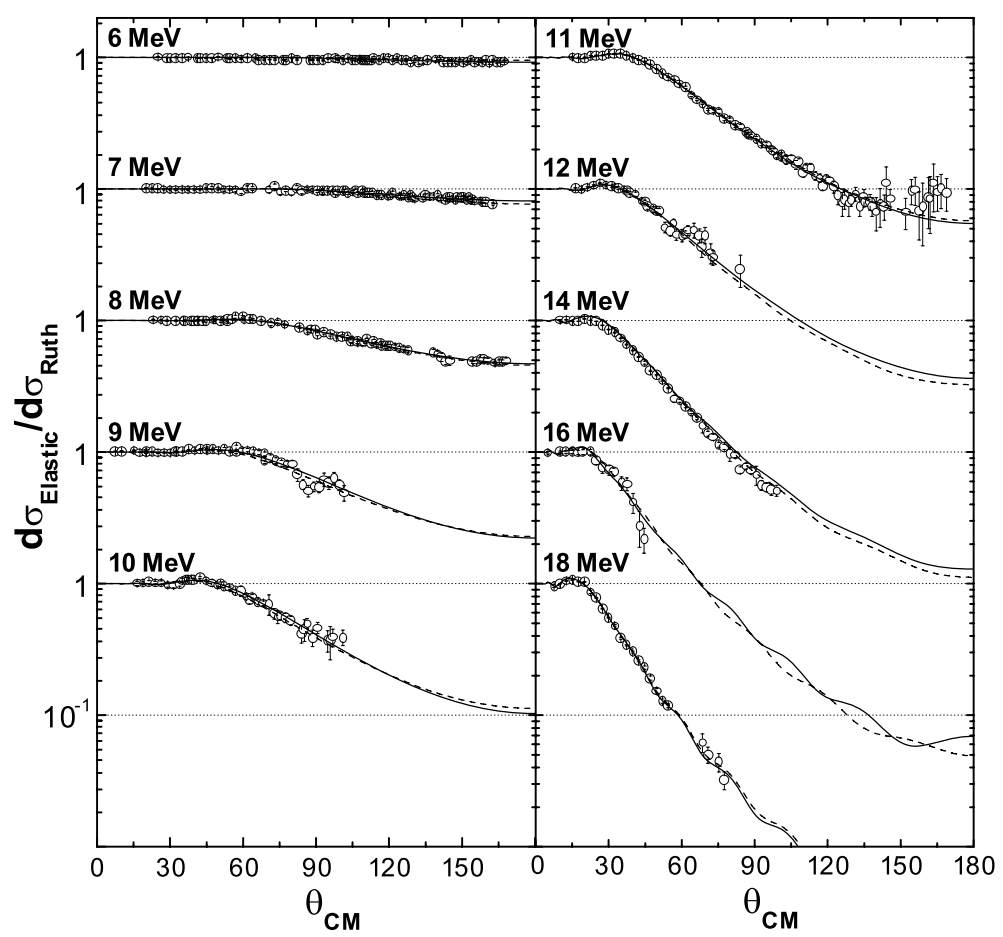

FIGURE 1. Elastic scattering cross sections normalized to the Rutherford cros sections for the ${ }^{7} \mathrm{Li}+$ ${ }^{27} \mathrm{Al}$ system and Optical Model calculations. Full lines correspond to an energy-dependent fit whereas the dashed lines correspond to an energy-independent one.

the values of the imaginary potential is almost constant except for the point at $6 \mathrm{MeV}$ which lies well above the rest. The real part of the potential at $6 \mathrm{MeV}$ has a larger error bar of about $4 \mathrm{MeV}$ due to the fact that the cross section at this energy is almost Rutherford.

In addition to the ${ }^{7} \mathrm{Li}+{ }^{27} \mathrm{Al}$ system, also the ${ }^{6} \mathrm{Li}+{ }^{27} \mathrm{Al}$ system has been measured. A preliminary analysis of these data have been performed and also fail to show the "normal threshold anomaly". Even more: the imaginary potential not only does not show any decrease at subbarrier energies but it shows a sharp increase, being in qualitative agreement with the so called break-up anomaly [8]. The complete analysis is underway and will be presented elsewhere.

\section{CONCLUSIONS}

In order to investigate threshold anomalies and to assess the relevance of the breakup channel in collisions of weakly bound nuclei we measured elastic scattering cross sections for the ${ }^{6,7} \mathrm{Li}+{ }^{27} \mathrm{Al}$ systems at energies around the Coulomb barrier. The present 


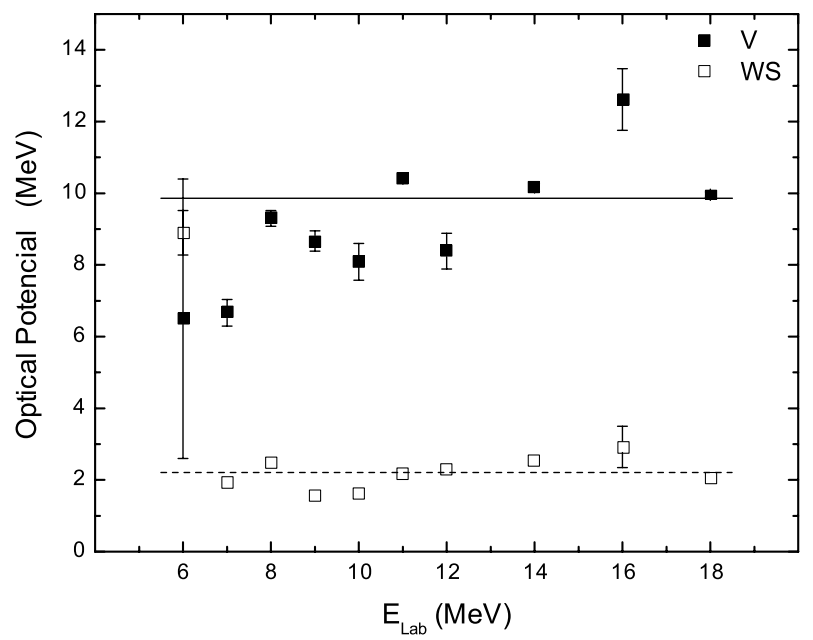

FIGURE 2. Depths of the real and imaginary potentials in energy-dependent (closed and open circles, respectively) and in energy-independent (solid and dashed lines, respectively) Optical Model fits.

analysis suggests no normal "threshold anomaly" or very weak one for the ${ }^{7} \mathrm{Li}+{ }^{27} \mathrm{Al}$ system. Further analysis with different potential shapes is under way. In the experimental point of view a direct measurement of the break up cross-section is being prepared.

\section{REFERENCES}

1. G. R. Satchler, Phys. Rep. 199, 147 (1991).

2. A. Pakou et al., Phys. Rev. C69, 054602 (2004).

3. A. Pakou et al., Phys. Lett. B556, 21 (2003).

4. E. de Barbará, contribution to this Proceedings.

5. M. H. MacFarlane et al., Argonne National Laboratory Report ANL-76-11, Rev. 1 (1983).

6. D. Abriola et al., Phys. Rev. C39, 546 (1989).

7. D. Abriola et al., Phys. Rev. C45, 244 (1992).

8. See for example L. F. Canto, P. R. S. Gomes, R. Donangelo, M. S. Hussein, Phys. Rep. 424, 1 (2006). 